\title{
Determination of the vertical electron-density profile in ionospheric tomography: experimental results
}

\author{
C. N. Mitchell, L. Kersley, J. A. T. Heaton, S. E. Pryse \\ The University of Wales, Aberystwyth, SY23 3BZ, UK Phone: + 4419706228 21, Fax: + 44197062 28 26, e-mail: cym@aber.ac.uk
}

Received: 12 June 1996 / Revised: 27 January 1997 / Accepted: 5 February 1997

\begin{abstract}
The reconstruction of the vertical electrondensity profile is a fundamental problem in ionospheric tomography. Lack of near-horizontal ray paths limits the information available on the vertical profile, so that the resultant image of electron density is biased in a horizontal sense. The vertical profile is of great importance as it affects the authenticity of the entire tomographic image. A new method is described whereby the vertical profile is selected using relative total-electroncontent measurements. The new reconstruction process has been developed from modelling studies. A range of background ionospheres, representing many possible peak heights, scale heights and electron densities are formed from a Chapman profile on the bottomside with a range of topside profiles. The iterative reconstruction process is performed on all of these background ionospheres and a numerical selection criterion employed to select the final image. The resulting tomographic images show excellent agreement in electron density when compared with independent verification provided by the EISCAT radar.
\end{abstract}

\section{Introduction}

Ionospheric tomography, proposed by Austen et al. (1986), has developed from initial modelling studies to being a viable technique for monitoring ionospheric electron density. The first experimental results were presented by Andreeva et al. (1990), while the first observation in which a tomographic image was verified by incoherent-scatter radar was reported by Pryse and Kersley (1992). Several experimental campaigns have been conducted since then: Kersley et al. (1993) showed promising results with the incorporation of ionosonde data into the reconstruction, and Foster et al. (1994) presented images from the Russian-American tomography experiment. A review of the experimental results has been written by Kersley and Pryse (1994), while a comparison of the different reconstruction algorithms can be found in Raymund (1995). More recently, Mitchell et al. (1995) showed tomographic images of a field-aligned boundary blob and the auroral-E region, the smallest verified structures which have been imaged by ionospheric tomography. Results presented by Pryse et al. (1995) show travelling ionospheric disturbances in consecutive tomographic images from data collected during December 1992. Markkanen et al. (1995) and Cook and Close (1995) have also demonstrated the tomographic imaging of travelling ionospheric disturbances. Experimental results from Russia and Finland (Kunitsyn et al., 1994) and recent images from Japan (Kunitake et al., 1995) demonstrate the increasing use of the tomographic technique to image ionospheric electron density.

For the reconstruction of a tomographic image in the ionospheric situation it is necessary to provide a priori information on the vertical electron-density profile (Yeh and Raymund, 1991). Lack of near-horizontal ray paths limits the information on the vertical profile available from the total-electron-content (TEC) measurements themselves. Several different approaches to the reconstruction have been pursued. Fremouw et al. (1992) used a set of orthonormal vectors, created from ionospheric models, to image the vertical electron-density profile. Sutton and $\mathrm{Na}$ (1994) have successfully implemented a similar technique called the orthogonal decomposition algorithm and demonstrated their method using simulated observations. Fehmers (1994) presented an algorithm which works independently from models, minimising on the basis of a number of constraints including vertical smoothness, horizontal flatness and upper and lower boundary conditions. A Bayesian statistical method has been tested on modelled and experimental data by Markannen et al. (1995). However, the ambiguity in the vertical electron-density profile proved to be a limitation to this approach.

Other reconstruction methods have relied on the vertical profile information provided by an ionospheric model (Raymund et al., 1993), such as IRI-90 (Bilitza, 1990), to create a background ionosphere with which to 
initialise an iterative algorithm. One possible solution to the problem was proposed by Heaton et al. (1995). The background bottomside ionosphere is created from the IRI-90 model and ionosonde data are incorporated to improve the representation of the peak height and the maximum electron density. The topside shape is selected from a linear combination of a library of normalised topside profiles. The method demonstrated an improvement in the reconstruction over earlier methods relying solely on the IRI-90 model for the background. There are, however, some disadvantages to this method. To use this method, the tomographic station chain needs to collocate with at least one ionosonde, but ionosondes are restricted in their geographic locations. Furthermore, certain ionospheric conditions render the ionosonde data unreliable; in particular at high latitudes blanketing auroral-E can prevent a signal echo from the $\mathrm{F}$ region. In addition, offvertical sounding from irregularities can cause an artificially high peak height to be inferred from the ionogram, which is then passed on to the tomographic image.

The new method contains two improvements over previous techniques. Firstly, the background does not have a peak height which has been fixed by a single model, since it involves the creation of models with a range of peak heights, scale heights, electron densities and topside shapes. Secondly, a new selection criterion is used to choose the image that best fits the tomographic data, after the reconstruction of all images has occurred. These two factors give the method some advantages. The peak height can be determined from the tomographic data, so that ionosonde information is no longer required. Furthermore, the selection of the vertical profile occurs after the reconstruction has been performed. Hence it is the reconstructed image that best fits the measurements, not the background, that defines the tomographic result.

\section{Experimental data}

Several experimental tomographic campaigns have been mounted in Scandinavia by the Aberystwyth group. Satellite receivers located along an approximate line of longitude have monitored radio transmissions from the Navy Navigational Satellite System (NNSS), satellites in polar orbits at altitudes of approximately $1100 \mathrm{~km}$. The reception of the phase coherent signals, transmitted from the satellites at 150 and $400 \mathrm{MHz}$, allows the derivation of the relative slant TEC along the satellite-to-receiver ray paths. The method to obtain slant TEC has been described by Kersley et al. (1993). Experimental data from two campaigns are presented in this paper. During March 1992 four receivers were located at Tromsø $\left(69.6^{\circ} \mathrm{N}, 19.2^{\circ} \mathrm{E}\right)$, Kiruna $\left(67.9^{\circ} \mathrm{N}, 20.4^{\circ} \mathrm{E}\right)$, Lycksele $\left(64.6^{\circ} \mathrm{N}, 18.8^{\circ} \mathrm{E}\right)$ and Uppsala $\left(59.8^{\circ} \mathrm{N}, 17.6^{\circ} \mathrm{E}\right)$, while the October 1993 campaign also used a fifth receiver at $\mathrm{Ny}$ Ålesund $\left(78.9^{\circ} \mathrm{N}, 12.0^{\circ} \mathrm{E}\right)$ on Svalbard.

The EISCAT radar at Tromsø, running in special programme mode, has been used to provide an independent comparison to the tomographic reconstructions. The special programme SP-UK-TOMO is desi- gned to image the electron-density structure in the auroral ionosphere for comparison with the tomographic images. In summary, the programme starts with the UHF radar beam observing in the zenith. The beam then scans southwards in the geographic meridian of Tromsø in steps of $0.125^{\circ}$ latitude at $300-\mathrm{km}$ altitude, each of 20-s duration. The duration of the complete scan is $20 \mathrm{~min}$, comparable to the horizon-to-horizon time of a satellite pass.

\section{Method}

The reconstruction of a tomographic image from limited angle data requires additional vertical profile information. A background ionosphere can be created to supply this initial information to the process, with the subsequent reconstruction of features relying on an iterative reconstruction algorithm (Raymund et al., 1993). In the method presented here relative TEC data are used in the iterative algebraic reconstruction technique (ART) (Gordon et al., 1970), with a weighting applied such that the modifications to a pixel are in proportion to its current density. This weighting is of importance as it ensures that the essential shape of the background profile is retained throughout the reconstruction. A broadly similar modification to the ART algorithm has been described by Andersen and Kak (1984).

The number of possible background ionospheres for use in any given experimental situation would run into many thousands, and the computing time could become prohibitive. In order to limit the number of reconstructions the method was performed as a two-stage process. The first stage was to produce estimates of the height and density of the layer peak, key parameters in the reconstructed image. A range of background ionospheres was produced using a Chapman profile with the following parameters: peak heights from 200 to $440 \mathrm{~km}$ in increments of $40 \mathrm{~km}$ and scale heights 60 to $120 \mathrm{~km}$ in increments of $20 \mathrm{~km}$. At this stage in the reconstruction there were 28 horizontally stratified background ionospheres. Considering one of these background ionospheres in matrix form, the initial electron density can be represented as $\boldsymbol{d}_{O_{N \times M}}$ for $N$ height and $M$ latitudinal pixels with the electron density normalised to that at the layer peak. An example of vertical profiles through two of these backgrounds can be seen in Fig. 1.

The peak electron density in the F-layer can vary by more than an order of magnitude. To span the entire range of possible densities a very large number of background ionospheres would be needed. Thus to limit the number of possible backgrounds, an initial estimate of the peak electron density at each latitude was taken from the IRI-90 model, represented by the diagonal of the matrix $\boldsymbol{I}_{p_{M \times M}}$. The electron-density profile, $\boldsymbol{x}_{o_{N \times M}}$, in the complete background ionosphere was then determined from:

$\boldsymbol{x}_{o_{N \times M}}=\boldsymbol{d}_{o_{N \times M}} \cdot \boldsymbol{I}_{p_{M \times M}} \cdot F$,

where $F$ is a further scaling factor which was varied from 0.2 to 1.8 in increments of 0.1 . An example of two 


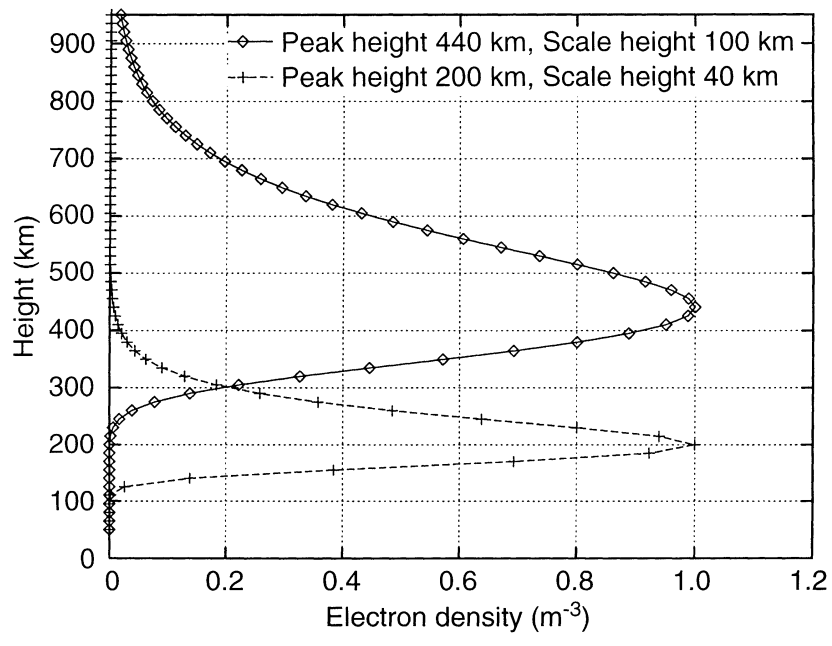

Fig. 1. Vertical electron-density profiles through two background ionospheres

extremes in background ionosphere is shown in Fig. 2. As the scaling was applied at all latitudes, there was a resultant latitudinal gradient in electron density from IRI-90 in each of the background ionospheres. In this way, the number of background ionospheres was increased by a factor determined by the number of peak densities (17). Taking into account all combinations of peak height (7), scale height (4) and peak density
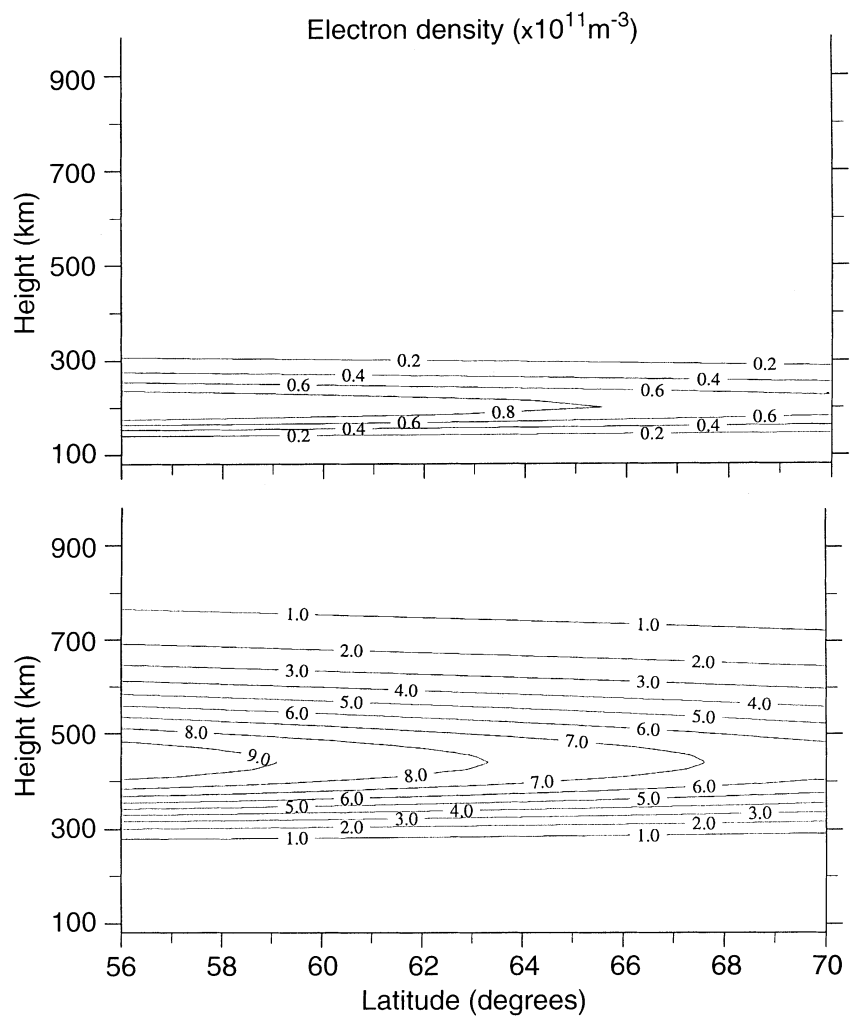

Fig. 2. Examples of background ionospheres used for the satellite pass at 13:15 UT on 17 October 1993. The upper plot was produced for a peak height of $200 \mathrm{~km}$ and a scale height of $60 \mathrm{~km}$. The density at each latitude was scaled by the peak density from IRI-90, then multiplied by a factor of 0.2 . The lower plot was produced for peak height $440 \mathrm{~km}$, scale height $100 \mathrm{~km}$ and a further density factor of 1.8
(17) there were now 476 background ionospheres. The first stage of the process was thus to reconstruct these 476 images and determine the one which best fitted the TEC data. This selection relied on the least-square error measurement, computing the relative TEC measurements and the relative TEC computation obtained from an integration along corresponding ray paths through the final image. The image which resulted in the smallest error was taken to provide an indication of the peak height and electron density.

Heaton et al. (1995) described an alternative approach to the representation of the topside shape in ionospheric tomography. Incoherent-scatter observations from both the UK and Scandinavia were used to provide a large database of topside shapes. By a process of averaging and normalisation these have been reduced to a number of shapes representing a wide range of ionospheric conditions. In the second stage of the current method the peak height and electron density can now be limited to a smaller range, making use of the information from the first stage. The described topside shapes are now attached to this subset of bottomside profiles to produce a new set of backgrounds. The reconstruction procedure is performed again on this new set of backgrounds with the range of topsides and the final selection of the tomographic image is again determined from the least-squares error.

\section{Results}

A satellite pass crossing $65^{\circ} \mathrm{N}$ at $13: 15$ UT was monitored by four Scandinavian receivers on the 17 October 1993 . The satellite's longitude at $65^{\circ} \mathrm{N}$ was $27^{\circ} \mathrm{E}$. The geomagnetic conditions were quiet with a $K_{\mathrm{p}}$ value of 1 . Co-ordinated observations using the EISCAT SP-UK-TOMO programme provided independent measurements of electron density to compare with the tomographic image. The EISCAT measurements from a scan starting at 13:00 UT are shown in Fig. 3a.

The first stage of the reconstruction for this pass indicated that the peak height was in the range 240 $280 \mathrm{~km}$. The second stage of the reconstruction involved a limited range of peak heights and densities with a larger number of topside shapes. Three peak heights were used: 230, 260 and $290 \mathrm{~km}$. The bottomside scale height was set to 50,60 or $70 \mathrm{~km}$, and 63 topside shapes were attached. In this way a large number of possible vertical profiles were provided as background ionospheres. The reconstruction was performed on all of these backgrounds and the tomographic image which best fitted the TEC data, when subjected to a leastsquares test, is shown in Fig. 3b. The EISCAT data provides an independent comparison to the tomographic image and it can be seen that excellent agreement has been achieved. The vertical profile through both images (Fig. 3c), taken at $66^{\circ} \mathrm{N}$, confirms that the correct selection has been made of the peak height and an excellent agreement in the vertical profile shape is apparent. It can be noted that the $30-\mathrm{km}$ vertical resolution chosen for the reconstruction corresponds 


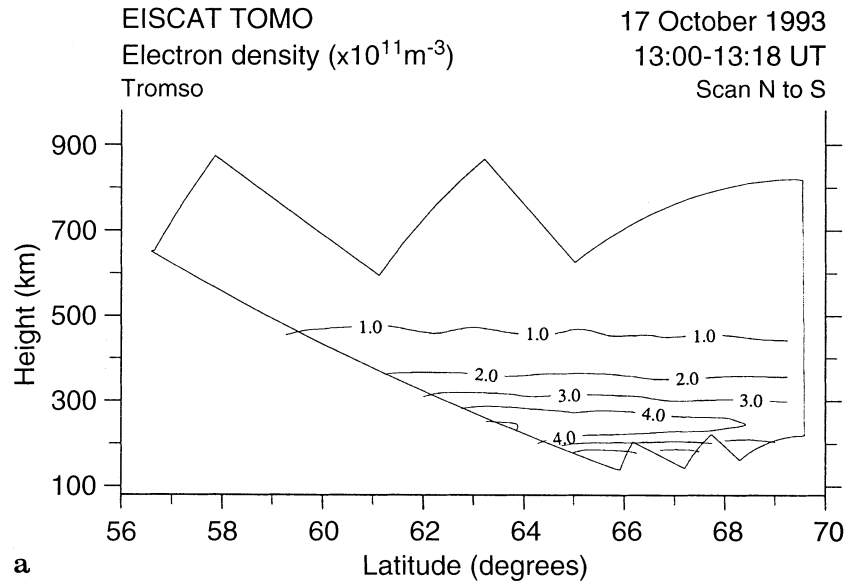

a

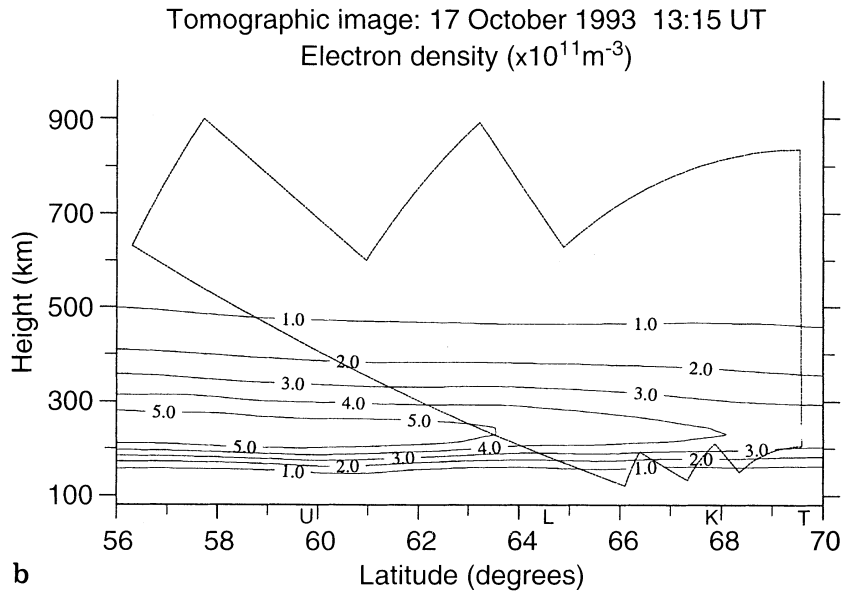

b

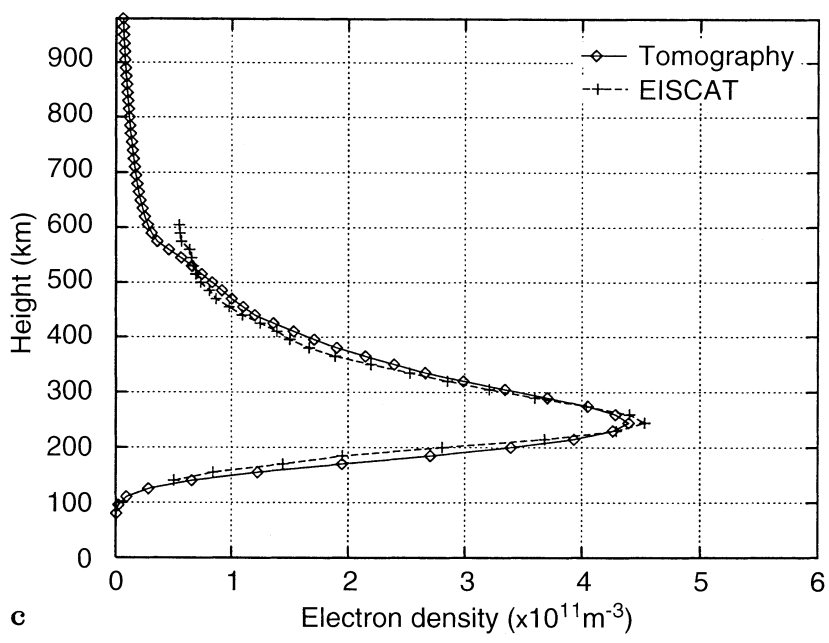

Fig. 3. a EISCAT SP-UK TOMO data from 13:00 to 13:18 UT, b tomographic image for a satellite pass at 13:15 UT on 17 October 1993 and c profiles through the images at $66^{\circ} \mathrm{N}$

to the pulse-pattern resolution of the radar. The RMS percentage electron-density difference between the tomographic image and the EISCAT data was $12 \%$, whereas the difference when the tomographic image was created from just an IRI-90 background was 19\%, showing the improvement achieved even for this example of an essentially horizontally stratified midday, midlatitude ionosphere. Further similar comparisons of reconstructions based on this new method indicated

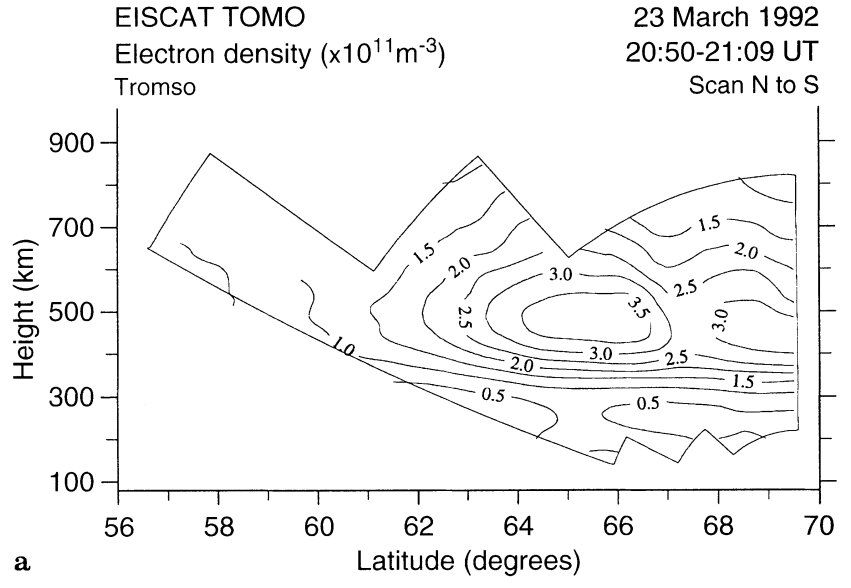

Tomographic image: 23 March 1992 20:58 UT Electron density $\left(\times 10^{11} \mathrm{~m}^{-3}\right)$

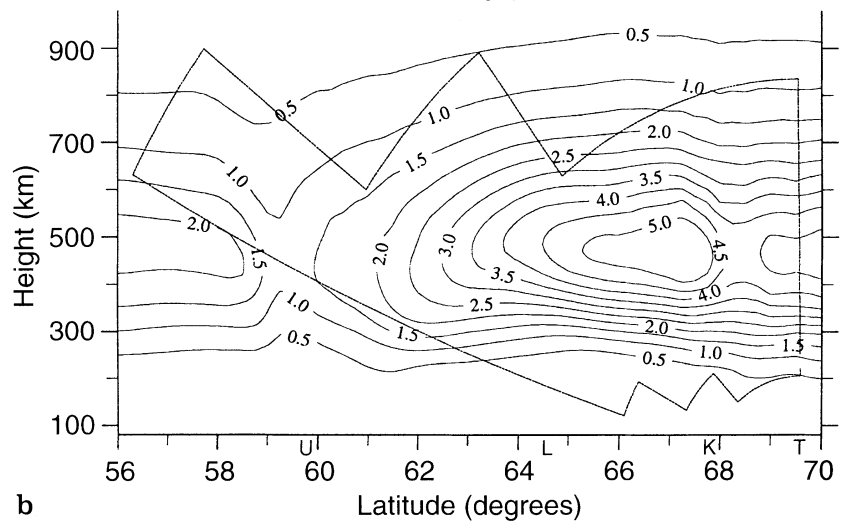

Fig. 4. a EISCAT SP-UK TOMO data from 20:50 to 21:09 UT and b tomographic image for a satellite pass at 20:58 UT on 23 March 1992

similar improvements over the use of the IRI-90 model to provide a background ionosphere. Using measurements from two other passes collected during a tomography campaign held in May 1995, a reduction in the RMS percentage electron-density difference between the tomographic image and SP-UK-TOMO data from EISCAT from $34 \%$ to $16 \%$ and from $37 \%$ to $13 \%$, respectively, was obtained from this method.

During the campaign of March 1992, a satellite pass crossing $65^{\circ} \mathrm{N}$ at 20:58 UT on 23 March provided data from very extreme ionospheric conditions on which to test the new method. The EISCAT measurements, shown in Fig. 4a, give the electron densities produced from a radar scan between 20:50 and 21:09 UT. Two enhancements in the electron density can be noted, centred on latitudes of $66^{\circ} \mathrm{N}$ and $69^{\circ} \mathrm{N}$. At the southern end of the scan the presence of a trough equatorward of $60^{\circ} \mathrm{N}$ is evident. The key feature is the height of the layer peak. It can be seen that the maximum electron density is found between some 400 to $500 \mathrm{~km}$, representing an extreme in ionospheric variability that presents a particular challenge to the imaging capabilities of ionospheric tomography.

The reconstructed tomographic image using the method described here is shown in Fig. $4 \mathrm{~b}$. For the 
reconstruction of this data set the peak heights in the background ionospheres were extended in range up to $540 \mathrm{~km}$, and the scale heights to $160 \mathrm{~km}$. The extreme peak height around $450 \mathrm{~km}$ has been correctly selected from the range of backgrounds. The two electrondensity enhancements and the trough minimum just below $60^{\circ} \mathrm{N}$ latitude have all been imaged by the tomographic technique. However, the densities at the layer peak are larger than those found by the radar. By taking a vertical electron-density profile through both the tomographic image and the EISCAT measurements at a latitude of $68.25^{\circ} \mathrm{N}$, shown as Reconstruction 1 in Fig. 5, the extent by which the tomography has overestimated the peak density becomes apparent. A closer inspection of the EISCAT profile shows evidence for an auroral-E electron-density enhancement, with an increase in density found below $200-\mathrm{km}$ altitude. The possibility of E-layer ionisation was not included in the range of vertical profiles submitted to the reconstruction process. Consequently, there is no enhancement in density below $200 \mathrm{~km}$ in the tomographic image. It is thus likely that the overestimation of the electron density at the $\mathrm{F}$ region has been caused, at least in part, by this E-region electron density being incorrectly placed at F-region heights by the reconstruction process. However, the tomographic image shown here with a layer maximum at a very high altitude represents a considerable improvement over the use of the IRI-90 model alone as a background. The RMS percentage difference, when tested against the EISCAT measurements, is improved by a factor of two when comparing the present method with the use of IRI-90 alone. However, at $64 \%$ this difference is still large, mainly due to the underlying auroral-E ionisation. Previous results given by Kersley et al. (1993) indicate that the use of a single model to provide a background ionosphere may be inappropriate in cases where the vertical profile departs significantly from that predicted by a model. The result presented here can be directly compared with that in Kersley et al. (1993). It can be seen that a

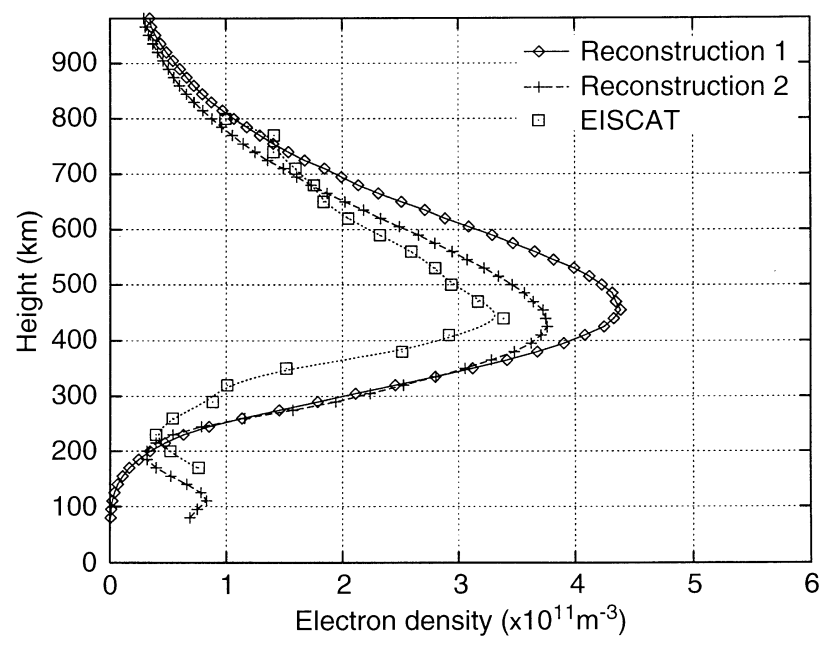

Fig. 5. Profiles through the tomographic images for a satellite pass at 20:58 UT on 23 March 1992 and EISCAT SP-UK TOMO data from 20:50 to $21: 09 \mathrm{UT}$ at $68.25^{\circ} \mathrm{N}$ significant improvement in the tomographic image has been achieved by the selection of the appropriate vertical profile.

The image was again reconstructed, now using bottomside profiles for the estimation of the background ionosphere that allowed for the possibility of a Chapman-type distribution of E-layer ionisation with a maximum at $110 \mathrm{~km}$. A profile through the resultant image at a latitude of $68.25^{\circ} \mathrm{N}$ is plotted as Reconstruction 2 in Fig. 5. It can be seen that while there is a marginal lowering of the peak F-layer height in this profile, it is comparable to that observed by the EISCAT radar. However, the magnitude of the electron density at the peak shows better agreement, with displacement of plasma into a substantial underlying E-layer. The peak density in the image now overestimates that measured by the radar by about $12 \%$. It should be noted that the calibration factor quoted for the radar at the time of this particular measurement represents an extreme value on the low side that deviates significantly within the set available from observations over many years. It is thus possible that the actual ionisation densities were in reality greater than those estimated here from the radar measurements. An increase of $10 \%$ in the calibration factor, which is well within the allowed range, would give an F-layer profile broadly comparable to that determined by tomography. However, in spite of this uncertainty in the absolute density, the imaging technique has been able to reproduce the height of the F-layer peak.

\section{Conclusions}

The new method has been demonstrated to produce tomographic reconstructions from experimental data which are verified by incoherent scatter, and in which images have been reconstructed without the use of ionosonde data or any other additional information to define the height of the layer peak. The tomographic image for 13:15 UT on 17 October 1993 demonstrates the authenticity that can be achieved by the tomographic technique. In this example, the vertical electrondensity profile was already well represented by the IRI-90 model, but use of the method described here resulted in a significant improvement in the residual error in the image. An RMS percentage error of only $12 \%$ was found over the region where the EISCAT radar provided an independent standard of comparison. However, the real test of the method was in its success in imaging a case where the ionospheric conditions displayed an extreme height, with a layer peak between 400 and $500 \mathrm{~km}$. The unexpectedly high peak height renders the use of a single ionospheric model unsuitable for creation of the background in the reconstruction process. Kersley et al. (1993) presented a reconstruction of this data set in which the USU model was used as a background. The resulting image had a peak height at $300 \mathrm{~km}$ and the residual RMS percentage difference from the EISCAT measurements was $377 \%$. A significant improvement in the determination of the peak 
height has been demonstrated in this present paper. The method is thus of particular importance in situations where the vertical profile of the ionosphere differs substantially from the median conditions represented by established ionospheric models. The need to consider auroral-E ionisation in establishing the form of the vertical profile in the high-latitude ionosphere has also been demonstrated. The initial work of Mitchell et al. (1995) illustrated the potential of the tomographic method in identifying the underlying ionisation in an auroral-E layer. The present work demonstrates that the F2-layer peak density may be overestimated unless provision is allowed in the reconstruction process for plasma at E-layer altitudes.

Acknowledgements. Thanks are due to the directors and staffs of the EISCAT Scientific Organisation at Tromsø and Kiruna, the Norsk Polarinstitutt at Ny Alesund, the Swedish Institute of Space Physics at Kiruna and Lycksele. The work was supported by a grant from the UK Particle Physics and Astronomy Research Council.

Topical Editor D. Alcaydè thanks J. Austen and A. Herique for their help in evaluating this paper.

\section{References}

Andersen, A. H., A. C. Kak, Simultaneous algebraic reconstruction technique (SART): a superior implementation of the art algorithm, Ultrason. Imaging, 6, 81-94, 1984.

Austen, J. R., S. J. Franke, C. H. Liu, and K. C. Yeh, Application of computerised tomography techniques to ionospheric research, Proc. Int. Beacon Satellite Symp., Univ. of Oulu, Finland, Ed. A. Tauriainen, pp 25-35, 1986.

Andreeva, E. S., A. V. Galinov, V. E. Kunitsyn, Yu. A. Mel'nichenko, E. D. Tereshchenko, M. A. Filimonov, and S. M. Chernykov, Radiotomographic reconstruction of ionisation dip in the plasma near the Earth, J. Exp. Theor. Phys. Lett., 52, 145-148, 1990.

Bilitza, D., International Reference Ionosphere 1990, National Space Science Data Center, Ref. 90-20, Greenbelt, Maryland, USA, 1990.

Cook, J. A., and S. Close, An investigation of TID evolution observed in MACE '93 data, Ann. Geophysicae, 13, 1320-1324, 1995.

Fehmers, G., A new algorithm for ionospheric tomography, Proc. Int. Beacon Satellite Symp., Univ. of Wales, Aberystwyth, Ed. L. Kersley, pp 52-55, 1994.

Foster, J. C., M. J. Buonsanto, J. M. Holt, J. A. Klobuchar, P. Fougere, W. Pakula, T. D. Raymund, V. E. Kunitsyn, E. S.
Andreeva, E. D. Tereshchenko, and B. Z. Khudukon, RussianAmerican tomography experiment, Int. J. Imaging Syst. Technol., 5, 148-159, 1994

Fremouw, E. J., J. A. Secan, and B. M. Howe, Application of stochastic inverse theory to ionospheric tomography, Radio Sci., 17, 721-732, 1992.

Gordon, R., R. Bender, and G. T. Herman, Algebraic reconstruction techniques (ART) for three-dimensional electron microscopy and X-ray photography, J. Theor. Biol., 29, 471-481, 1970.

Heaton, J. A. T., S. E. Pryse, and L. Kersley, Improved background representation, ionosonde input and independent verification in experimental ionospheric tomography, Ann. Geophysicae, 13, 1297-1303, 1995.

Kersley, L., and S. E. Pryse, Development of experimental ionospheric tomography, Int. J. Imaging Syst. Technol., 5, 141-147, 1994.

Kersley, L., J. A. T. Heaton, S. E. Pryse, and T. D. Raymund, Experimental ionospheric tomography with ionosonde input and EISCAT verification, Ann. Geophysicae, 11, 1064-1074, 1993.

Kunitake, M., K. Ohtaka, T. Maruyama, M. Tokamaru, A. Morioka, and S. Watanabe, Tomographic imaging of the ionosphere over Japan by the modified truncated SVD method, Ann. Geophysicae, 13, 1303-1310, 1995.

Kunitsyn, V. E., E. S. Andreeva, E. D. Tereshchenko, B. Z. Khudukon, and T. Nygrén, Investigations of the ionosphere by satellite radiotomography, Int. J. Imaging Syst. Technol., 5, 112-127, 1994.

Markkannen, M., M. Lehtinen, T. Nygrén, J. Pirtilä, P. Henelius, E. Vilenious, E. D. Tereshchenko, and B. Z. Khudokon, Bayesian approach to satellite radiotomography with applications in the Sandinavian sector, Ann. Geophysicae, 13, 1277-1287, 1995.

Mitchell, C. N., D. G. Jones, L. Kersley, S. E. Pryse, and I. K. Walker, Imaging of field-aligned structures in the auroral ionosphere, Ann. Geophysicae, 13, 1311-1319, 1995.

Pryse, S. E., and L. Kersley, A preliminary experimental test of ionospheric tomography, J. Atmos. Terr. Phys., 54, 1007-1012, 1992.

Pryse, S. E., C. N. Mitchell, J. A. T. Heaton, and L. Kersley, Tomographic imaging of travelling ionospheric disturbances, Ann. Geophysicae, 13, 1325-1331, 1995.

Raymund, T. D., Comparison of several ionospheric tomography algorithms, Ann. Geophysicae, 13, 1254-1262, 1995.

Raymund, T. D., S. E. Pryse, L. Kersley, and J. A. T. Heaton, Tomographic reconstruction of the ionospheric electron density with EISCAT verification, Radio Sci., 28, 811-817, 1993.

Sutton, E., and H. Na, Orthogonal decomposition framework for ionospheric tomography algorithms, Int. J. Imag. Syst. Technol., 5, 106-111, 1994.

Yeh, K. C., and T. D. Raymund, Limitations of ionospheric imaging by tomography, Radio Sci., 26, 1361-1380, 1991. 